\title{
Improving the Biocompatibility of Polymeric Membrane Potentiometric Ion Sensors by Using a Mussel-Inspired Polydopamine Coating
}

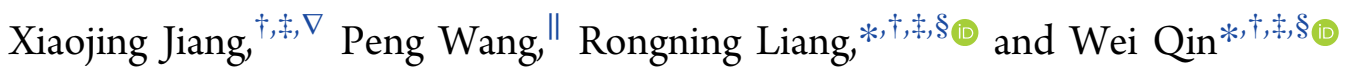 \\ ${ }^{\dagger}$ CAS Key Laboratory of Coastal Environmental Processes and Ecological Remediation, Yantai Institute of Coastal Zone Research, \\ Chinese Academy of Sciences, Yantai, Shandong 264003, People's Republic of China \\ ${ }^{\ddagger}$ Shandong Key Laboratory of Coastal Environmental Processes, Yantai Institute of Coastal Zone Research, Chinese Academy of \\ Sciences, Yantai, Shandong 264003, People's Republic of China \\ ${ }^{\S}$ Laboratory for Marine Biology and Biotechnology, Qingdao National Laboratory for Marine Science and Technology, Qingdao \\ 266200, People's Republic of China \\ ${ }^{\nabla}$ University of Chinese Academy of Sciences, Beijing 100049, People's Republic of China \\ "Shandong B\&E Bio-technology Co., Ltd, Yantai, People's Republic of China
}

\section{Supporting Information}

ABSTRACT: Polymeric membrane potentiometric ion sensors have been widely used in clinical diagnosis for the detection of electrolyte ions and account for billions of measurements every year throughout the world. However, in many cases of practical relevance, biofouling, which might lead to sensor failure, usually occurs due to the lack of biocompatibility of these sensors. Herein, we describe a simple and robust approach for improving the biocompatibility of the polymeric ionselective membranes. A marine mussel-inspired polydopamine polymer is used as a hydrophilic coating on the surface of conventional potentiometric ion sensors. Such a coating can be easily formed by self-polymerization of dopamine and robustly deposited on the sensor surface mimicking the adhesion mechanism of mussels. The classical poly(vinyl chloride) membrane-based calcium ion-selective electrode (ISE) is chosen as a model. Compared to the unmodified $\mathrm{Ca}^{2+}$ ISE, the polydopamine modified electrode shows a significantly reduced blood platelet adsorption while retaining original potentiometric ion response properties, which clearly indicates a high antifouling capability induced by the hydrophilic polydopamine coating. We believe that the proposed approach can provide an appealing way to improve the biocompatibility in the development of polymeric membrane electrochemical and optical sensors.
$\mathrm{P}$ olymeric membrane ion-selective electrodes (ISEs) are well-established analytical tools and have been widely utilized in a variety of fields, from clinical analysis to environmental monitoring. ${ }^{1-5}$ In clinical applications especially, potentiometry with polymeric membrane ISEs has become the standard technique in the clinical analysis of electrolyte ions such as $\mathrm{Na}^{+}, \mathrm{K}^{+}, \mathrm{Ca}^{2+}$, and $\mathrm{Cl}^{-}$. Many clinical analyzers based on ISEs have been developed and are commercially available for monitoring analytes in the clinical samples such as whole blood, serum, and urine in clinical laboratories and at the bedsides of critically ill patients. However, note that reliable determination of ions in these biological samples is only feasible by using flow-through ISEbased sensors. This is mainly because biofouling problems such as the adsorptions of proteins and lipids onto the sensor surface usually exist when these sensors are continuously in contact with these samples. $^{6-8}$ In particular, for in vivo analysis, e.g., implantable sensors for long-term monitoring of blood analytes, biofouling can become a serious issue. The adsorption of proteins on surfaces of implantable sensors can lead to a series of biological responses, such as the coagulation, cell adhesion, and eventual thrombus formation. Such blood clots may affect the electrochemical performance of an implantable sensor by perturbing the analyte mass transfer to the sensor surface, and further cause measurement errors, because of the metabolic byproducts of adsorbed cells. ${ }^{7,9}$

Several approaches have been proposed to reduce the effects of biofouling of polymeric membrane potentiometric sensors. ${ }^{10}$ One promising approach to improve the blood compatibility of these sensors is based on the continuous release of biologically active molecules such as nitric oxide (NO) from the sensing membrane to the sample, ${ }^{11}$ which can considerably decrease the platelet activation. Although this approach is very effective

Received: January 3, 2019

Accepted: April 29, 2019

Published: April 29, 2019 
in biocompatibility improvement of polymeric membrane ISEs, it may be limited by the sensor lifetime since NO releasing materials in the membrane are continuously consumed. Other approaches are to modify the ISE membranes to obtain antifouling properties, including the preparation of ISE membranes with surface-immobilized anticoagulants such as heparin, $^{12}$ and grafting biocompatible polymers such as poly(ethylene oxide), ${ }^{7}$ poly(ethylene glycol) ${ }^{9}$ and tetraethylene glycol $^{13}$ on the outer surface of the membrane. Although these approaches were successful in enhancing the biocompatibility of the ISE membranes, they are usually involved in complicated synthesis or modification procedures. It has been reported that biocompatibility may be increased by simply coating with hydrophilic materials, ${ }^{14}$ which can increase the surface hydrophilicity of ISE membranes. Hydrophilic coatings of a hydrogel, poly(2-hydroxyethyl methacrylate) (pHEMA), ${ }^{15}$ and a copolymer, 2-methacryloyloxyethyl phosphorylcholine (MPC) and n-butyl or 2-ethylhexyl methacrylate, ${ }^{8,16}$ have been employed to improve biocompatibility. Unfortunately, these coatings can easily peel off from the sensing membrane surface, because of the poor adhesion between the hydrophilic coating and the surface of the polymer membrane.

Herein, a simple and robust approach is proposed to improve the biocompatibility of polymeric membrane ISEs based on a marine mussel-inspired polydopamine coating. ${ }^{17}$ The adhesive proteins of mussels contain unusually high concentrations of catechol and amine functional groups, which are capable of mediating adhesions to most organic and inorganic surfaces. Recently, Messersmith and co-workers reported a robust surface coating by using dopamine as a small-molecule mimic of the adhesive proteins. ${ }^{18}$ Such a coating can deposit on virtually any substrate surface by simple immersion of the substrate in dopamine solution at a weakly alkaline $\mathrm{pH}$. Moreover, the coating on the substrate surface has good stability and durability in various environments, because some covalent and noncovalent interactions, such as the hydrogen bonding, $\pi-\pi$, and electrostatic interactions, may exist between polydopamine and the substrate. ${ }^{19,20}$ Inspired by this breakthrough, as well as several examples of using polydopamine in surface hydrophilic modification in materials science, ${ }^{21,22}$ we have developed a facile approach to increase the hydrophilicity of polymeric membrane potentiometric sensors, thereby improving the biocompatibility of such sensors. In this work, poly(vinyl chloride) (PVC)-based polymeric membrane $\mathrm{Ca}^{2+}$-ISE has been chosen as a model of potentiometric sensors. The surface hydrophilic modification is simply achieved by immersion of the sensor in a dopamine solution at $\mathrm{pH}$ 8.5. Experimental results show that the proposed approach offers a simple but robust way to fabricate highly biocompatible polymeric membrane potentiometric sensors.

\section{MATERIALS AND METHODS}

Materials. High-molecular-weight PVC, $o$-nitrophenyl octyl ether (o-NPOE), sodium tetrakis[3,5-bis (trifluoromethyl) phenyl]borate (NaTFPB), the ionophore ( $N, N$-dicyclohexyl$N^{\prime}, N^{\prime}$-dioctadecyl-3-oxapentanediamide (ETH 5234), dopamine hydrochloride, poly(sodium 4-styrenesulfonate), (NaPSS, molecular weight $(\mathrm{Mw})$ of 70000$)$, 3,4-ethylenedioxythiophene (EDOT, 97\%), heparin sodium salt (from bovine intestinal mucosa, 149 units/mg), and bovine serum albumin (BSA) were purchased from Sigma-Aldrich. Aqueous solutions were prepared with freshly deionized water (specific resistance $=18.2 \mathrm{M} \Omega \mathrm{cm}$ ) obtained with a Pall Cascada laboratory water system. Tetrahydrofuran (THF) was freshly distilled prior to use. All other reagents were analytical grade and used without any further purification. A stock solution of $0.01 \mathrm{M} \mathrm{Ca}^{2+}$ was prepared by dissolving $\mathrm{CaCl}_{2}$ in deionized water and then diluted to various concentrations of working solutions with deionized water prior to measurements.

Preparation of Solid-Contact $\mathrm{Ca}^{2+}$-ISEs. The membrane cocktail containing ETH 5234 (0.8 wt \%), NaTFPB (0.4 wt \%), o-NPOE (65.8 wt \%), and PVC (33.0 wt \%) was prepared by dissolving $360 \mathrm{mg}$ membrane components in 3.6 $\mathrm{mL}$ of THF.

The solid-contact ISEs based on poly(3,4-ethylenedioxythiophene) (PEDOT)-PSS were prepared following the previously reported procedures. ${ }^{23}$ Briefly, PEDOT was electrodeposited on the polished glassy carbon (GC) electrode with a diameter of $3 \mathrm{~mm}$ by galvanostatic electrochemical polymerization in a deaerated aqueous solution containing $0.01 \mathrm{M}$ EDOT and 0.1 M NaPSS as the supporting electrolyte. A constant current was applied to produce a polymerization charge of $10 \mathrm{mC}$ in the polymerization process. The prepared electrode (GC/PEDOT-PSS) was washed with deionized water and conditioned with $0.001 \mathrm{M} \mathrm{CaCl}_{2}$ for at least $24 \mathrm{~h}$. Then, $90 \mu \mathrm{L}$ of the membrane cocktail was drop-cast onto the GC/PEDOT-PSS electrode and left to dry overnight at room temperature.

Hydrophilic Modification. Hydrophilic modification of the electrodes was achieved by simple immersion of the solidcontact ISEs obtained above in a dopamine solution $(20 \mathrm{mg} /$ $\mathrm{mL}$ in $10 \mathrm{mM}$ Tris buffer solution, $\mathrm{pH} 8.5$ ) for $12 \mathrm{~h}$. After polymerization, the obtained electrodes were thoroughly washed with deionized water. For detection of $\mathrm{Ca}^{2+}, 0.001$ $\mathrm{M} \mathrm{CaCl} 2$ was used as the conditioning solution, while for evaluation of the selectivity coefficients, the conditioning solution was $0.01 \mathrm{M} \mathrm{NaCl}$. All electrodes were conditioned in a solution identical to the conditioning solution for $12 \mathrm{~h}$ before measurements.

Contact Angle Measurements. The contact angles of PVC sensing membranes were measured with an optical microscope equipped with a goniometer ocular. The contact liquid used was distilled water and the volume of the droplet was $10 \mu \mathrm{L}$. Drops of the testing liquid were deposited by a glass syringe onto the electrode surfaces and the contact angles were measured after a defined period of time in order to allow the establishment of equilibrium.

Electrochemical Measurements. All measurements of electromotive force (EMF) were performed at room temperature, using a PXSJ-216 pH meter (Leici, Shanghai, PRC) in the galvanic cell: saturated calomel electrode (SCE)//0.1 M $\mathrm{LiOAc/sample} \mathrm{solution/ISE} \mathrm{membrane/PEDOT-PSS/GC}$ electrode. The external reference electrode employed was a double-junction SCE with $0.1 \mathrm{M} \mathrm{LiOAc}$ as a bridge electrolyte. Selectivity coefficients were determined by a separate solution method. $^{24}$ The EMF values were corrected for the liquidjunction potentials with the Henderson equation. The activity coefficients of ions were calculated from the modified DebyeHückel equation. $^{25}$

Platelet Adhesion Studies. Blood samples were obtained from the carotid arteries of the healthy duck and chicken from the local market. After collection, an anticoagulant solution (heparin sodium salt, 149 units/mg) was added to blood to reach a final concentration of $0.1 \mathrm{~g} / \mathrm{L}$. The platelet-rich plasma 
Scheme 1. Schematic Illustration of One-Step Hydrophilic Conversion of the PVC Membrane ISE, Based on Mussel-Inspired Self-Polymerization of Dopamine

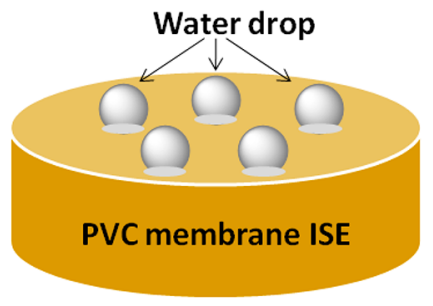

Hydrophobic membrane sensor

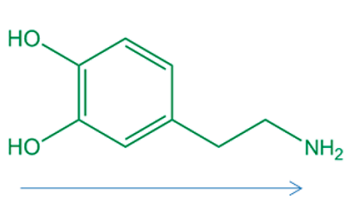

One-step selfpolymerization

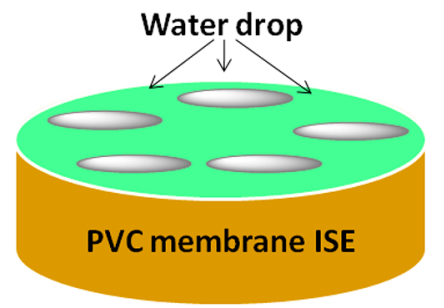

Hydrophilic membrane sensor
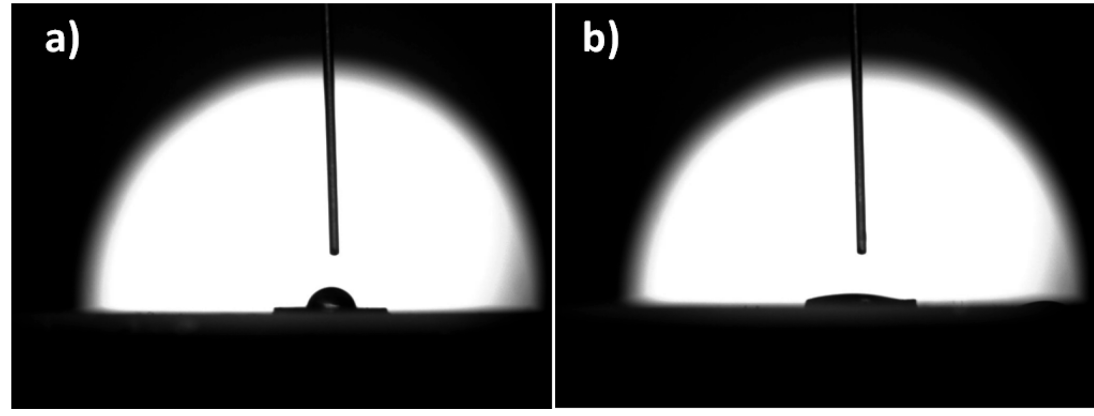

Figure 1. Contact angles of the water drops for (a) the classical unmodified and (b) polydopamine-modified PVC Ca ${ }^{2+}$-selective membranes.

(PRP) supernatant was obtained by centrifugation of blood at $1800 \mathrm{rpm}$ for $20 \mathrm{~min}$. The preconditioned polymer membrane sensing electrodes were immersed in PRP for $2 \mathrm{~h}$ at room temperature. They were then gently rinsed with $0.01 \mathrm{M}$ phosphate buffer saline (PBS) of $\mathrm{pH} 7.4$ for three times (25 $\mathrm{mL}$ for $5 \mathrm{~s}$ once; the related explanations can be found in the Supporting Information). For potentiometric measurements, the resultant electrodes were transferred to a cell containing aqueous $\mathrm{CaCl}_{2}$ standards. For graphic evaluation of the deposited platelets on the sensor surface, these electrodes were fixed in a PBS solution containing $2.0 \%(\mathrm{w} / \mathrm{v})$ glutaraldehyde for $2 \mathrm{~h}$ in order to immobilize the adsorbed platelets on membrane surface. The obtained membranes were subsequently dehydrated by immersion in serial dilutions of ethanol $(30,50,70,90,95,100 \% \mathrm{v} / \mathrm{v})$. After desiccation with a infrared light, the membranes were coated with gold and characterized utilizing field-emission scanning electron microscopy (FE-SEM) (Model S-4800, Hitachi, Japan).

Potentiometric Detection in Whole Blood Samples. The freshly collected blood samples were first added with the anticoagulant solution and then directly transferred to a separate electrochemical cell containing the working ISE electrode and the reference electrode for potentiometric detection. As a comparison, the commercially available electrolyte analyzer (Model CBS-400, Shandong B\&E Biotechnology Co., Ltd.) was used as the standard technique for evaluation of the amounts of $\mathrm{Ca}^{2+}$ in blood samples.

\section{RESULTS AND DISCUSSION}

During the past decades, several strategies have been proposed to improve the biocompatibility of polymeric membrane ISEs in order to make them reliable for continuous blood sample analysis and even the eventual monitoring in vivo. ${ }^{10}$ These attempts have made great contributions toward the improvement of the biocompatibility, but the developed sensors are usually involved with complicated synthesis processes or unstable surface modification layers. This poses serious limits to their wide use, especially for long-term in vivo monitoring. Hence, in this work, a simple but robust method is described to improve the biocompatibility of the ISEs.

Since PVC is the most frequently used matrix to prepare polymer membrane-type ISEs, the PVC-based $\mathrm{Ca}^{2+}$-ISE was chosen as a model. The proposed robust surface modification coating was formed simply by one-step immersion of the $\mathrm{Ca}^{2+}$ ISE in a dopamine solution with a $\mathrm{pH}$ of 8.5 , which is based on the self-polymerization of dopamine and the strong adhesion of polydopamine. ${ }^{18}$ Such adhesion might be ascribed to some noncovalent interactions between the PVC membrane and polydopamine, such as the $\pi-\pi$ interaction between the phenyl groups of polydopamine and $o-\mathrm{NPOE}$, and the hydrogen bonding interaction between the hydroxyl group of polydopamine and the nitro group of $o$-NPOE. As illustrated, the hydrophobic surface of the membrane electrode can be easily converted to the hydrophilic one (Scheme 1). Since the polymerization time and the dopamine concentration play vital roles in the thickness and hydrophilicity of the obtained polydopamine layer, ${ }^{26}$ the influences of both parameters were investigated. The contact angle measurements of water droplets were performed to characterize the surface hydrophilicity of the PVC sensing membrane. The results are shown in Figures S1 and Figure S2, respectively, in the Supporting Information. As shown in Figure S1, the water contact angle decreases rapidly as the concentration of dopamine solution increases up to $20 \mathrm{mg} / \mathrm{mL}$, which is due to the increase in the number of hydrophilic groups, such as amine and hydroxyl groups, on the surface of the PVC membrane. On the other hand, longer polymerization times can also result in a thicker polydopamine layer, thus leading to a more hydrophilic surface layer. Hence, it can be naturally predicted that the hydrophilicity would be improved with increasing the polymerization time. Indeed, the PVC sensing membrane has a smaller contact angle as the time increases up to $12 \mathrm{~h}$ and further increases in 
the polymerization time would not obviously improve the hydrophilicity (see Figure S2). The results of the contact angle measurements for the PVC-based $\mathrm{Ca}^{2+}$-ISEs before and after polydopamine modification with $20 \mathrm{mg} / \mathrm{mL}$ dopamine for $12 \mathrm{~h}$ are shown in Figure 1. As illustrated, the hydrophobic membrane surface becomes hydrophilic with a dramatic decrease in the contact angle from $77.9^{\circ} \pm 3.5^{\circ}$ to $33.5^{\circ} \pm$ $1.5^{\circ}$ after polydopamine functionalization. Indeed, polydopamine can be effectively used to improve the surface hydrophilicity of the PVC-based ISE.

Note that the improvement in sensor biocompatibility should not influence the analytical performance. ${ }^{7}$ In view of this, the modified and unmodified PVC membrane $\mathrm{Ca}^{2+}$-ISEs were tested in the concentration range of $10^{-8}-10^{-2} \mathrm{M}$. The results are shown in Figure 2. It can be seen that no significant

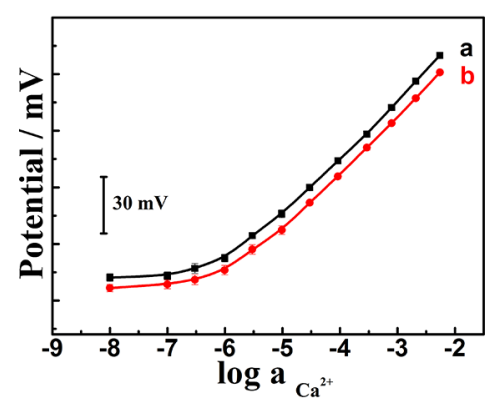

Figure 2. Potentiometric responses of (a) unmodified and (b) polydopamine-modified polymeric membrane $\mathrm{Ca}^{2+}$-ISEs. Each data point represents an average \pm standard deviation for three measurements.

difference could be observed between potentiometric responses of both electrodes, which indicates that the polydopamine modification has little effect on the electrode performance of the polymeric membrane $\mathrm{Ca}^{2+}$-ISEs. Indeed, the response slope and the detection limit remain almost the same. The unmodified electrode exhibits a Nernstian response of $29.3 \mathrm{mV} /$ decade over the concentration range of $10^{-6}-10^{-2} \mathrm{M}$ with a detection limit of $7.1 \times 10^{-7} \mathrm{M}$, while the modified electrode shows a similar Nernstian slope $(28.3 \mathrm{mV} /$ decade $)$ and detection limit $\left(5.8 \times 10^{-7} \mathrm{M}\right)$ as well. In addition, the experiments also show that no $\mathrm{pH}$ dependence was introduced into our proposed sensing system (see Figure S3 in the Supporting Information), since the sensing membrane is the PVC-based $\mathrm{Ca}^{2+}$-ISE membrane but not the polydopamine layer. However, the response time of the modified electrode appears to be significantly longer than that of the classical unmodified one (see Figure 3). Such change in the response time can be attributed to the fact that the modification layer may influence the ion diffusion by kinetically reducing $\mathrm{Ca}^{2+}$ ion fluxes in the aqueous layer. ${ }^{27}$ Notably, the proposed hydrophilic layer may affect the ion diffusion but not impair the final thermodynamic extraction equilibrium state of $\mathrm{Ca}^{2+},{ }^{28-30}$ which can be confirmed by their similar response properties, in terms of linear range and detection limit. The observed unchanged characteristics are probably due to the thin nature of the polydopamine coating and the weak $\mathrm{Ca}^{2+}$-chelating abilities of the phenolic hydroxyl and secondary amine groups in the coating. In this case, the polydopamine layer cannot change the concentration of $\mathrm{Ca}^{2+}$ at the membrane surface diffused from the sample bulk to the surface of the polymeric

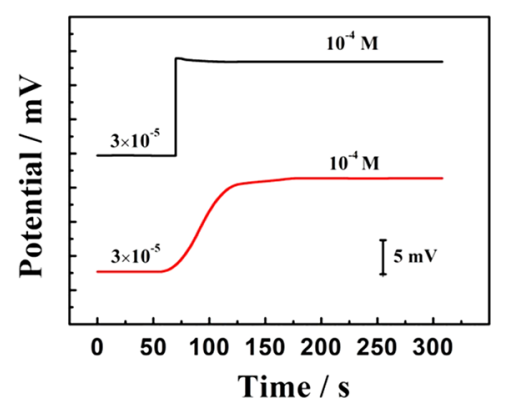

Figure 3. Typical dynamic potential responses with time of (upper) the classical unmodified and (lower) polydopamine-modified PVCbased $\mathrm{Ca}^{2+}$-ISEs when the $\mathrm{Ca}^{2+}$ concentration was changed from $3 \times$ $10^{-5} \mathrm{M}$ to $10^{-4} \mathrm{M}$.

membrane. Thus, the sensor still responds to the concentration of $\mathrm{Ca}^{2+}$ in the bulk of the sample. ${ }^{29}$

The selectivity coefficients of the $\mathrm{Ca}^{2+}$-selective membranes for interfering ions were determined by using Bakker's method to eliminate the influence of the inherent sensitivity limit on the ISE responses toward discriminated ions. ${ }^{24}$ Table S1 in the Supporting Information compares the selectivity coefficients of the modified and unmodified $\mathrm{Ca}^{2+}$-selective membranes. As can be seen, nearly all potentiometric selectivity coefficients of modified membrane are comparable to those of the classical unmodified membrane. Thus, it can be demonstrated that the hydrophilic modification does not influence the selectivity coefficients based on the thermodynamic equilibrium response, further indicating that the modification cannot affect the extraction equilibrium state of $\mathrm{Ca}^{2+}$.

In order to illustrate the biocompatibility of the proposed hydrophilic $\mathrm{Ca}^{2+}$-ISE, both modified and unmodified electrodes were evaluated by using the classical platelet adhesion test, which is considered to be helpful as an initial screening for selecting biocompatible materials. ${ }^{7,11}$ All electrodes were left in the PRP solution for $2 \mathrm{~h}$ at room temperature to allow for platelets adsorption prior to potentiometric measurements. The results are shown in Figure 4. Clearly, the polydopamine-

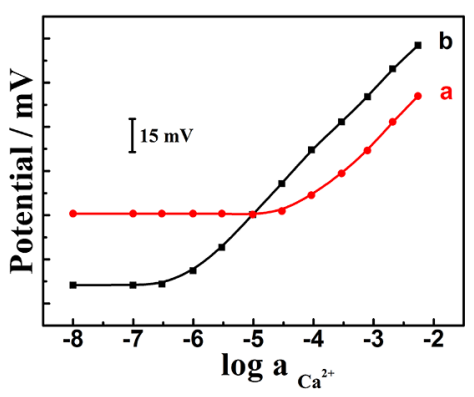

Figure 4. Typical calibration curves of (a) classical unmodified and (b) modified hydrophilic PVC membrane $\mathrm{Ca}^{2+}$ electrodes after contact with PRP solution for $2 \mathrm{~h}$.

modified electrode differs significantly from the unmodified one in the key sensing parameters, such as slope and detection limit after contact with the PRP solution. The response behaviors of the modified $\mathrm{Ca}^{2+}$ electrode are almost unchanged (curve $\mathrm{b}$ in Figure 4 versus curve $\mathrm{b}$ in Figure 2), while the unmodified classical electrode suffers from a serious interference problem from platelet adsorption (curve a in Figure 4). These results indicate that the proposed hydrophilic $\mathrm{Ca}^{2+}$-ISE shows an improved fouling resistance. The modified electrode 


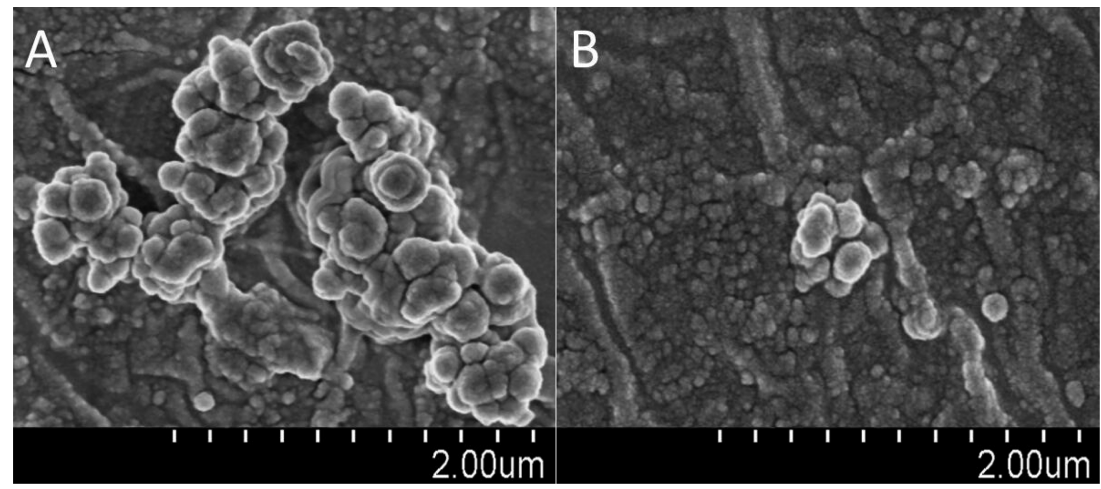

Figure 5. SEM images of (a) unmodified and (b) modified PVC-based $\mathrm{Ca}^{2+}$ membranes after $2 \mathrm{~h}$ contact with PRP solution.

exhibits a wider linearity range of $10^{-6}-10^{-2} \mathrm{M}$, a better Nernst slope of $27.8 \mathrm{mV} /$ decade, and a lower detection limit of $5.6 \times 10^{-7} \mathrm{M}$, compared to the unmodified one, with a rather poor detection limit of $4.8 \times 10^{-5} \mathrm{M}$. Such a remarkable difference is probably due to the fact that the unmodified hydrophobic PVC membrane has a tendency to promote conformational changes of proteins in blood upon contact, thus resulting in a higher packing density of adsorbed molecules, while the hydrophilic surface of polydopaminemodified electrode can prevent the adsorption of hydrophobic blood proteins. ${ }^{14,31}$ When the layer of blood proteins on a PVC-sensing membrane surface becomes thick enough, the electrode responds to the concentration of $\mathrm{Ca}^{2+}$ within the adsorbed layer, instead of the $\mathrm{Ca}^{2+}$ concentration in the sample. $^{29}$ In this case, the attainable detection limit of unmodified electrode would be deteriorated.

Furthermore, the membranes were characterized via SEM to evaluate the amounts of deposited blood cells before and after contact with the PRP solution. As illustrated, before contact, the unmodified membrane shows a flat and homogeneous surface (Figure S2a) while the modified one exhibits a rough appearance, because of the polydopamine coating (Figure $\mathrm{S} 2 \mathrm{~b})$. After contact, the classical unmodified sensing membrane has a large number of deposited platelets with densely packed platelet aggregates (Figure 5), indicating that the unmodified membrane is potentially thrombogenic. ${ }^{32}$ In contrast, a significant decrease in platelet adhesion is observed for the polydopamine-modified hydrophilic membrane. These results further confirm that the biocompatibility of PVC-based $\mathrm{Ca}^{2+}$-ISE is indeed improved by the polydopamine modification. Such excellent biocompatibility of the proposed $\mathrm{Ca}^{2+}$-ISE offers great potential for potentiometric detection in real biological samples.

Also, the proposed electrode has a good reproducibility and long-term stability for $\mathrm{Ca}^{2+}$ detection. For $10^{-4} \mathrm{M} \mathrm{Ca}^{2+}$, the relative standard deviation (RSD\%) was found to be $4.1 \%$ for one electrode with six measurements and $6.5 \%$ for six electrodes prepared with identical procedures. Only a $1.7 \%$ change in the Nernstian slope of the proposed ISE was observed after storage at $4{ }^{\circ} \mathrm{C}$ for 1 month.

In order to illustrate its accuracy in practical analysis, the comparison between the proposed hydrophilic sensor and unmodified sensor for detection of $\mathrm{Ca}^{2+}$ in real samples was performed. The results are shown in Table S2 in the Supporting Information. It can be seen that the data obtained by the hydrophilic sensor agree well with those obtained by the commercial electrolyte analyzer. However, note that the $\mathrm{Ca}^{2+}$ concentrations are usually high in animal blood samples (i.e., $1.5 \mathrm{mM}$ ), so that the conventional unmodified PVC $\mathrm{Ca}^{2+}$-ISE could also function well in blood samples (see curve a in Figure 4). Hence, to further illustrate the excellent antibiofouling ability of the proposed hydrophilic sensor, both electrodes were applied to detect $\mathrm{Ca}^{2+}$ ions in samples containing low levels of $\mathrm{Ca}^{2+}$ and a high protein background. The results are summarized in Table S3 in the Supporting Information. As predicated, the proposed hydrophilic sensor can be reliably used to detect trace-level $\mathrm{Ca}^{2+}$ ions in the presence of a high protein background, while the data obtained by the unmodified PVC-based sensor seriously deviate from the real values, indicating that the hydrophilic sensor shows an excellent antibiofouling ability. Such difference can be attributable to the hydrophilic modification, which significantly inhibits the protein adsorption, thus improving the biocompatibility of PVC sensing membrane. These results suggest that the proposed sensor has promising feasibility for determination of trace-level $\mathrm{Ca}^{2+}$ in complex samples.

\section{CONCLUSIONS}

In this work, a simple and robust method for improving the biocompatibility of PVC-based polymeric membrane potentiometric ion sensors is proposed for the first time. The method is based on a marine mussel-inspired hydrophilic polydopamine coating, which can be tightly deposited on virtually any substrate surface. The proposed modification is achieved by simply immersion of the PVC membrane ISE in a dopamine solution at $\mathrm{pH}$ 8.5. Note that, although Fragoso et al. ${ }^{33}$ reported a potentiometric $\mathrm{pH}$ sensing electrode based on the similar polydopamine coating, their $\mathrm{pH}$ electrode is not a polymeric membrane ISE. Their polydopamine layer was formed directly on a solid-contact-based glassy carbon electrode substrate. In this case, their sensing membrane was the polydopamine layer but not the PVC $\mathrm{Ca}^{2+}$-selective membrane employed in this work. In addition, no biocompatibility study was involved in Fragoso's work. In our work, compared to the classical unmodified ISE, the proposed polydopamine-modified electrode exhibits significantly improved biocompatibility for dramatically reduced blood component adhesion and an excellent sensing ability in complex biological samples. In view of the simple, robust, and general nature of the polydopamine modification layer, this new approach can be extended to improve the biocompatibilities of other materials-based electrochemical and optical sensors for the analysis of biological samples. 


\section{ASSOCIATED CONTENT}

\section{S Supporting Information}

The Supporting Information is available free of charge on the ACS Publications website at DOI: 10.1021/acs.analchem.9b00039.

Influence of the concentration of dopamine on the contact angle of PVC membrane $\mathrm{Ca}^{2+}$-ISE (Figure S1); effect of polymerization time of dopamine on the contact angle of PVC membrane $\mathrm{Ca}^{2+}$-ISE (Figure S2); potential responses of the proposed polydopaminemodified $\mathrm{Ca}^{2+}$-ISE to $10^{-4} \mathrm{M} \mathrm{Ca}^{2+}$ at different $\mathrm{pH}$ levels (Figure S3); SEM images of unmodified and polydopamine modified PVC-based $\mathrm{Ca}^{2+}$ membranes before contact with PRP solution (Figure S4); potentiometric selectivity coefficients obtained with the separate solution method for unmodified and modified PVC membrane $\mathrm{Ca}^{2+}$-ISEs (Table S1); comparison of the results obtained by the unmodified and polydopamine (Table S2); comparison of the results obtained by the unmodified and polydopamine-modified PVC Ca ${ }^{2+}$-ISEs in laboratory-prepared samples containing a low level of $\mathrm{Ca}^{2+}$ and a high concentration of protein (Table S3); explanations for the recovery of the baseline potential of the proposed electrode in the presence of the polydopamine modification layer (PDF)

\section{AUTHOR INFORMATION}

\section{Corresponding Authors}

*Fax: +86-535-2109000. E-mail: rnliang@yic.ac.cn (R. Liang). *Fax: +86-535-2109000. E-mail: wqin@yic.ac.cn (W. Qin).

\section{ORCID $\odot$}

Rongning Liang: 0000-0002-9693-1242

Wei Qin: 0000-0002-9606-7730

Notes

The authors declare no competing financial interest.

\section{ACKNOWLEDGMENTS}

This work was financially supported by the National Natural Science Foundation of China (Nos. 21874151, 21677172, 41576106), the National Key Research and Development Program of China (No. 2016YFC1400700), the Youth Innovation Promotion Association of CAS (No. 2014190) and the Taishan Scholar Program of Shandong Province (No. tspd20181215).

\section{REFERENCES}

(1) Zdrachek, E.; Bakker, E. Anal. Chem. 2019, 91, 2-26.

(2) Bobacka, J.; Ivaska, A.; Lewenstam, A. Chem. Rev. 2008, 108, 329-351.

(3) Chen, L. D.; Mandal, D.; Pozzi, G.; Gladysz, J. A.; Bühlmann, P. J. Am. Chem. Soc. 2011, 133, 20869-20877.

(4) Meyerhoff, M. E.; Fu, B.; Bakker, E.; Yun, J. H.; Yang, V. C. Anal. Chem. 1996, 68, 168A-175A.

(5) Papp, S.; Jágerszki, G.; Gyurcsányi, R. E. Angew. Chem., Int. Ed. 2018, 57, 4752-4755.

(6) Lisak, G.; Arnebrant, T.; Lewenstam, A.; Bobacka, J.; Ruzgas, T. Anal. Chem. 2016, 88, 3009-3014.

(7) Espadas-Torre, C.; Meyerhoff, M. E. Anal. Chem. 1995, 67, $3108-3114$

(8) Berrocal, M. J.; Johnson, R. D.; Badr, I. H. A.; Liu, M. D.; Gao, D. Y.; Bachas, L. G. Anal. Chem. 2002, 74, 3644-3648.

(9) Pawlak, M.; Grygolowicz-Pawlak, E.; Crespo, G. A.; Mistlberger, G.; Bakker, E. Electroanalysis 2013, 25, 1840-1846.
(10) Pawlak, M.; Bakker, E. Electroanalysis 2014, 26, 1121-1131.

(11) Espadas-Torre, C.; Oklejas, V.; Mowery, K.; Meyerhoff, M. E. J. Am. Chem. Soc. 1997, 119, 2321-2322.

(12) Brooks, K. A.; Allen, J. R.; Feldhoff, P. W.; Bachas, L. G. Anal. Chem. 1996, 68, 1439-1443.

(13) Pawlak, M.; Mistlberger, G.; Bakker, E. J. Mater. Chem. 2012, 22, 12796-12801.

(14) Ekdahl, K. N.; Lambris, J. D.; Elwing, H.; Ricklin, D.; Nilsson, P. H.; Teramura, Y.; Nicholls, I. A.; Nilsson, B. Adv. Drug Delivery Rev. 2011, 63, 1042-1050.

(15) Murphy, S. M.; Hamilton, C. J.; Davies, M. L.; Tighe, B. J. Biomaterials 1992, 13, 979-990.

(16) Yajima, S.; Sonoyama, Y.; Suzuki, K.; Kimura, K. Anal. Chim. Acta 2002, 463, 31-37.

(17) Lee, H.; Lee, B. P.; Messersmith, P. B. Nature 2007, 448, $338-$ 341 .

(18) Lee, H.; Dellatore, S. M.; Miller, W. M.; Messersmith, P. B. Science 2007, 318, 426-430.

(19) Lee, H.; Scherer, N. F.; Messersmith, P. B. Proc. Natl. Acad. Sci. U. S. A. 2006, 103, 12999.

(20) Bernsmann, F.; Ponche, A.; Ringwald, C.; Hemmerlé, J.; Raya, J.; Bechinger, B.; Voegel, J. C.; Schaaf, P.; Ball, V. J. Phys. Chem. C 2009, 113, 8234-8242.

(21) Liu, X. S.; Cao, J. M.; Li, H.; Li, J. Y.; Jin, Q.; Ren, K. F.; Ji, J. ACS Nano 2013, 7, 9384-9395.

(22) Kang, S. M.; You, I.; Cho, W. K.; Shon, H. K.; Lee, T. G.; Choi, I. S.; Karp, J. M.; Lee, H. Angew. Chem., Int. Ed. 2010, 49, 9401-9404.

(23) Bobacka, J. Anal. Chem. 1999, 71, 4932-4937.

(24) Bakker, E. J. Electrochem. Soc. 1996, 143, L83-L85.

(25) Kamata, S.; Bhale, A.; Fukunaga, Y.; Murata, H. Anal. Chem. 1988, 60, 2464-2467.

(26) McCloskey, B. D.; Park, H. B.; Ju, H.; Rowe, B. W.; Miller, D. J.; Chun, B. J.; Kin, K.; Freeman, B. D. Polymer 2010, 51, 3472-3485.

(27) Muslinkina, L.; Pretsch, E. Chem. Commun. 2004, 1218-1219.

(28) Xu, Y. D.; Xu, C.; Shvarev, A.; Becker, T.; De Marco, R.; Bakker, E. Anal. Chem. 2007, 79, 7154-7160.

(29) Boswell, P. G.; Bühlmann, P. J. Am. Chem. Soc. 2005, 127, 8958-8959.

(30) Ceresa, A.; Pretsch, E.; Bakker, E. Anal. Chem. 2000, 72, 20502054.

(31) Norde, W. Adv. Colloid Interface Sci. 1986, 25, 267-340.

(32) Lindner, E.; Cosofret, V. V.; Buck, R. P.; Johnson, T. A.; Ash, R. B.; Neuman, M. R.; Kao, W. J.; Anderson, J. M. Electroanalysis 1995, 7, 864-870.

(33) Zuaznabar-Gardona, J. C.; Fragoso, A. Sens. Actuators, B 2018, 273, 664-671. 